\title{
CRB3 Binds Directly to Par6 and Regulates the Morphogenesis of the Tight Junctions in Mammalian Epithelial Cells
}

\author{
Céline Lemmers, ${ }^{*}$ Didier Michel, ${ }^{*}$ Lydie Lane-Guermonprez, ${ }^{*}$ \\ Marie-Hélène Delgrossi, Emmanuelle Médina, Jean-Pierre Arsanto, and \\ André Le Bivic ${ }^{\dagger}$
}

\begin{abstract}
Laboratoire de Neurogenèse et Morphogenèse au cours du Développement et chez l'Adulte, Unité Mixte Recherche 6156 Centre National de la Recherche Scientifique, Institut de Biologie du Développment de Marseille, Université de la Méditerranée, Campus de Luminy, 13288 Marseille, France
\end{abstract}

Submitted April 16, 2003; Revised September 26, 2003; Accepted October 24, 2003

Monitoring Editor: Mark Ginsberg

\begin{abstract}
Crumbs is an apical transmembrane protein crucial for epithelial morphogenesis in Drosophila melanogaster embryos. A protein with all the characteristics for a Crumbs homologue has been identified from patients suffering from retinitis pigmentosa group 12, but this protein (CRB1) is only expressed in retina and some parts of the brain, both in human and mouse. Here, we describe CRB3, another Crumbs homologue that is preferentially expressed in epithelial tissues and skeletal muscles in human. CRB3 shares the conserved cytoplasmic domain with other Crumbs but exhibits a very short extracellular domain without the EGF- and laminin A-like G repeats present in the other Crumbs. CRB3 is localized to the apical and subapical area of epithelial cells from the mouse and human intestine, suggesting that it could play a role in epithelial morphogenesis. Indeed, expression of CRB3 or of a chimera containing the extracellular domain of the neurotrophin receptor p75NTR and the transmembrane and cytoplasmic domains of CRB3 led to a slower development of functional tight junctions in Madin-Darby canine kidney cells. This phenotype relied on the presence of CRB3 four last amino acids (ERLI) that are involved in a direct interaction with Par6, a regulator of epithelial polarity and tight junction formation. Thus, CRB3, through its cytoplasmic domain and its interactors, plays a role in apical membrane morphogenesis and tight junction regulation.
\end{abstract}

\section{INTRODUCTION}

The acquisition of polarity in epithelial cells is a fundamental mechanism crucial for the development of multicellular organisms. This process involves transmembrane proteins acting as cell surface organizers that nucleate cortical proteins, thus providing a scaffold for the building of junctional complexes that physically separate the apical surface from the basolateral domain of the plasma membrane (Knust, 2000; Tepass et al., 2001). Sorting of newly synthesized proteins is then necessary to maintain the polarized distribution of apical and basolateral proteins (Mostov et al., 2000; Nelson and Yeaman, 2001). Cell surface organizers such as integrins and cadherins have been identified in mammalian epithelial cells (Yeaman et al., 1999), but in the last few years, genetics in Drosophila proved to be instrumental in opening new perspectives on protein complexes involved in epithelial polarity (Muller, 2000). Among such complexes, the Crumbs complex stands out because it contains an apical transmem-

Article published online ahead of print. Mol. Biol. Cell 10.1091/ mbc.E03-04-0235. Article and publication date are available at www.molbiolcell.org/cgi/doi/10.1091/mbc.E03-04-0235.

* These authors contributed equally to this work.

${ }^{+}$Corresponding author. E-mail address: lebivic@ibdm.univ-mrs.fr. Abbreviations used: MDCK, Madin-Darby kidney cell; NTR, neurotrophin receptor; PATJ, protein associated to tight junctions; PDZ, PSD95/Discs large/ZO-1; PRC, photoreceptor cell; TJ, tight junction. brane protein crucial for cell morphogenesis (Rashbass and Skaer, 2000; Tepass et al., 2001). Crumbs is a large (2139-aa) glycoprotein with an extracellular domain containing 30 EGF-like repeats and four laminin A-like G domains, a transmembrane domain, and a short cytoplasmic domain of 37 amino acids (Tepass et al., 1990). Expression of $c r b$ starts at gastrulation, and the protein is accumulated at the subapical region (marginal zone) of ectodermal epithelial cells where it colocalizes with two other gene products involved in epithelial polarity, Stardust (sdt) and Discs lost (dlt). These three genes, $c r b$, sdt, and $d l t$, are essential for the development of the ectodermal layer of the embryos, and their loss of function leads to a failure in the correct assembly of the zonula adherens (ZA) and epithelial disorganization (Grawe et al., 1996; Bhat et al., 1999; Bachmann et al., 2001; Hong et al., 2001). In fact, recent studies have shown that Crumbs binds directly through the last amino acids (ERLI) of its cytoplasmic tail to Stardust, a protein of the membrane-associated guanylate kinase family (Bachmann et al., 2001; Hong et al. 2001). This complex recruits Discs lost, a protein containing four PDZ (PSD95/Discs large/ZO-1) domains (Bhat et al., 1999) building a scaffold in the marginal zone. The marginal zone also contains another scaffolding complex made of at least three proteins Par3 (bazooka)/Par6/atypical protein kinase $C$, and it has been proposed that the two complexes interact by using genetic experiments (Bilder et al., 2003; Tanentzapf and Tepass, 2003). Recently, several studies have shown that Crumbs is also involved in the morphogenesis of photoreceptors (PRCs) in Drosophila (Izaddoost et al., 2002; 
Johnston and Gallant, 2002; Pellikka et al., 2002). This is of particular importance because it was shown that a homologue of $c r b$, named CRB1, was mutated both in patients suffering from retinitis pigmentosa group 12 (den Hollander et al., 1999) and Leber congenital amaurosis (den Hollander et al., 2001). These two pathologies are characterized by PRC loss and retinal degeneration, supporting the hypothesis that CRB1 and Crumbs are key elements for PRC morphogenesis and function in mammals and in Drosophila, respectively. CRB1 is, however, not expressed in human epithelial cells (den Hollander et al., 2002), and the question remains whether another homologue of $c r b$ is present in these cells and plays a role in controlling apical morphogenesis.

In this report, we have studied a human Crumbs, called CRB3. CRB3 is a small transmembrane protein with a conserved cytoplasmic domain containing the two motives GTY and ERLI found in Crumbs (Klebes and Knust, 2000). CRB3 exhibits an additional Src homology type 3 binding site (PxxP) not found in other Crumbs. CRB3 is mainly expressed in epithelial tissues and skeletal muscles. Using specific antibodies, we showed that CRB3 localizes to the apical membrane and the apical part of the junctional complex of epithelial cells of mouse intestine. Overexpression of human CRB3 and of a chimera made of p75NTR extracellular domain and transmembrane and cytoplasmic domains of CRB3 in Madin-Darby canine kidney (MDCK) cells did not disrupt dramatically apicobasolateral polarity but led to a defect in the establishment and stability of tight junctions (TJs). This effect was dependent upon the presence of the ERLI motif, and we identified Par6, a major component involved in epithelial polarity and asymmetric divisions, as a direct interactor of the cytoplasmic tail of CRB3. These data indicate that CRB3, together with Par6, plays a role in regulating the structure of the apical membrane and tight junctions.

\section{MATERIALS AND METHODS}

\section{Reagents and Antibodies}

Protein A-Sepharose and glutathione-Sepharose were from Pharmacia AB (Uppsala, Sweden). Monoclonal antibody (mAb) against occludin was from Zymed Laboratories (South San Francisco, CA) and was used at a dilution of 1:200 for immunofluorescence, whereas mAb 9E10 against myc was from Santa Cruz Biotechnology (Santa Cruz, CA). A mouse monoclonal ME 20-4 against human p75NTR (Le Bivic et al., 1991), BC11 against a basolateral antigen, and BC44 against an apical antigen of MDCK cells (Beau et al., 1998) were used at a dilution of 1:200 for immunofluorescence. Polyclonal antibodies against GP114 and PATJ were described previously (Le Bivic et al., 1990; Lemmers et al., 2002) and polyclonal antibodies against ZO-1 and -3 were a kind gift from K. Matter (University College London, London, United Kingdom). Polyclonal antibodies against the cytoplasmic domain of CRB3 were produced by the injection of purified histidine-tagged cytoplasmic domain into rabbits $(75 \mu \mathrm{g} /$ boost, a boost every $3 \mathrm{wk})$ and used at a 1:500 dilution for immunofluorescence on cells, 1:200 dilution for immunofluorescence on tissue sections, 1:25 for immunogold on ultrathin frozen sections, and 1:50 for Western blots. A polyclonal antibody raised against the last amino acids of hCRB3 (a gift of B. Margolis, Howard Hughes Medical Institute, Ann Arbor, MI) was also used 1:800 for blotting experiments. Antibodies against Par6 were a gift from W.J. Nelson (Stanford University School of Medicine, Palo Alto, CA).

\section{Constructs, Cell Culture, and Transfection}

hCRB3 cDNA was cloned by reverse transcription-polymerase chain reaction (RT-PCR) by using the superscript kit (Invitrogen, Carlsbad, CA) and Caco-2 total RNAs, respectively. PCR amplification was performed with High Fidelity Polymerase (Roche Diagnostics, Meylan, France). His-tagged CRB3 was obtained by subcloning the full cytoplasmic domain of hCRB3 in pQE-30 vector from QIAGEN (Valencia, CA) and expression in M15 Escherichia coli, according to the manufacturer's instructions. The cDNA fragments encoding the cytoplasmic domain of hCRB3 (+ / - ERLI) or the PDZ domain of hPar6 $\alpha$ were subcloned into pGEX 4 T3 to produce glutathione $S$-transferase (GST) fusion proteins according to the manufacturer (Pharmacia AB). To test antiCRB3 sera specificity, constructs were designed by replacing the cytoplasmic domain of dCrumbs with either CRB1, 2, or 3 cytoplasmic domains in a previously described construct containing the VSV-G tag, the stalk region, transmembrane domain, and cytoplasmic domain of dCrumbs (amino acids 2074-2146) (Lemmers et al., 2002). This fusion construct was subsequently subcloned into the EcoRI-BamHI sites of pIRES1neo vector (BD Biosciences Clontech, Palo Alto, CA). Chimeras between the human receptor for neurotrophins, p75NTR, and hCRB3 were performed by PCR by using human p75NTR cDNA subcloned into pIRES and human CRB3 cDNA isolated by RT-PCR. All constructs were fully sequenced and tested for expression in COS-7 and MDCK cells by using FuGENE 6 (Roche, Diagnostics).

\section{Northern Blots and Western Blots}

Northern blots were performed as described previously (Delgrossi et al., 1997) by using human poly(A) RNA from BD Biosciences Clontech) and the coding sequence of human CRB3 as a probe (Delgrossi et al., 1997). Western blots on COS-7 cells transfected with VSV-G-tagged cytoplasmic domains of CRB1, 2, or 3, or MDCK cells expressing hCRB3 or myc-tagged Par6 constructs were processed as published previously (Lemmers et al., 2002) by using sera against CRB3 or the anti-myc 9E10 antibody. Membrane preparations from MDCK cells were obtained as described previously (Lemmers et al., 2002).

\section{Two-Hybrid Screen, GST Pull-Downs, and Coimmunoprecipitations}

A human breast library from BD Biosciences Clontech in pACT2 was used to identify new partners of the cytoplasmic domain of hCRB3 inserted in the pBTM116 vector. Then, $10^{6}$ transformed (growing in the absence of tryptophane and leucine) clones were plated, and $\sim 200$ clones were harvested after selection in the absence of tryptophane, leucine, and histidine and in the presence of $10 \mathrm{mM} 3$-aminotriazole. Clones were sorted by their digestion pattern. The majority (75\%) were identified as hPar6 $\alpha$ after sequencing. For GST pull-downs, 1\% Triton X-100 extract of COS-7 cells transfected with myc-tagged full-length Par6 or its C-terminal part was incubated overnight with glutathione-Sepharose beads $(30 \mu \mathrm{l})$ coated with GST-hCRB3 cytoplasmic domain fusion protein $(40 \mu \mathrm{g})$ and after washes, bound proteins were analyzed by Western blotting after SDS-PAGE. For coimmunoprecipitations, MDCK cells expressing human p75NTR, p75-CRB3, or p75-CRB3- $\Delta$ ERLI were processed as described in Hurd et al. (2003).

\section{Immunofluorescence, Confocal Microscopy, and Immunogold Labeling of Cells and Tissues}

Cells were grown on glass coverslips or transwell filters (Costar, Corning, NY) and processed as described previously (Le Bivic et al., 1988), whereas sections of mouse intestine were labeled as described in Lemmers et al. (2002). Confocal microscopy analysis was performed using an LSM 510 confocal microscope (Carl Zeiss, Jena, Germany). For immunoelectron microscopy, ultrathin frozen sections of human colon were obtained and processed as described previously (Delgrossi et al., 1997) by using goat anti-rabbit antibodies coupled to 6-nm gold particles (Saito et al., 2001). Standard transmission electron microscopy on cells grown into Matrigel was performed as described in Lipschutz et al. (2000).

\section{Matrigel Culture, Calcium Switch, and Epithelial Integrity Assays}

MDCK cells expressing human p75NTR, p75-CRB3, or p75-CRB3- $\Delta$ ERLI were grown into Matrigel gels (final concentration $60 \%$ ), layered on glass coverslips (for 7-10 d) or polycarbonate filters (Transwell; Costar) (for 10-20 d), and treated for immunofluorescence as described in Lipschutz et al. (2000). Calcium removal was obtained by washing twice the cells with phosphatebuffered saline containing $2 \mathrm{mM}$ EGTA and maintaining them in a $\mathrm{Ca}^{2+}$-free medium (Gao et al., 2002) for the time of the experiment. To measure the tightness of the monolayers formed by stably transfected MDCK cells, cells were seeded at confluence on polycarbonate filters (Transwell; Costar). After 12 or $24 \mathrm{~h}$ of culture, $\left[{ }^{3} \mathrm{H}\right]$ inulin $(110,000 \mathrm{cpm} / \mathrm{ml})$ was added in the apical compartment and after $1 \mathrm{~h}$ of incubation at $37^{\circ} \mathrm{C}$, the amount of radioactivity present in the apical and the basolateral compartment was measured using a beta counter (1450 Microbeta Plus; PerkinElmer Wallac, Turku, Finland).

\section{RESULTS}

\section{CRB3 Is Preferentially Expressed in Epithelial Tissues}

Although there is only one crumbs gene in $D$. melanogaster, in human and mouse, three genes, CRB1, 2, and 3, have been identified, and all of them are expressed because their products are found in expressed sequence tag (EST) databases (Medina et al., 2002a). It is now a major challenge to understand the role of each CRB in mammalian development and physiology. In particular, their potential role in epithelial 


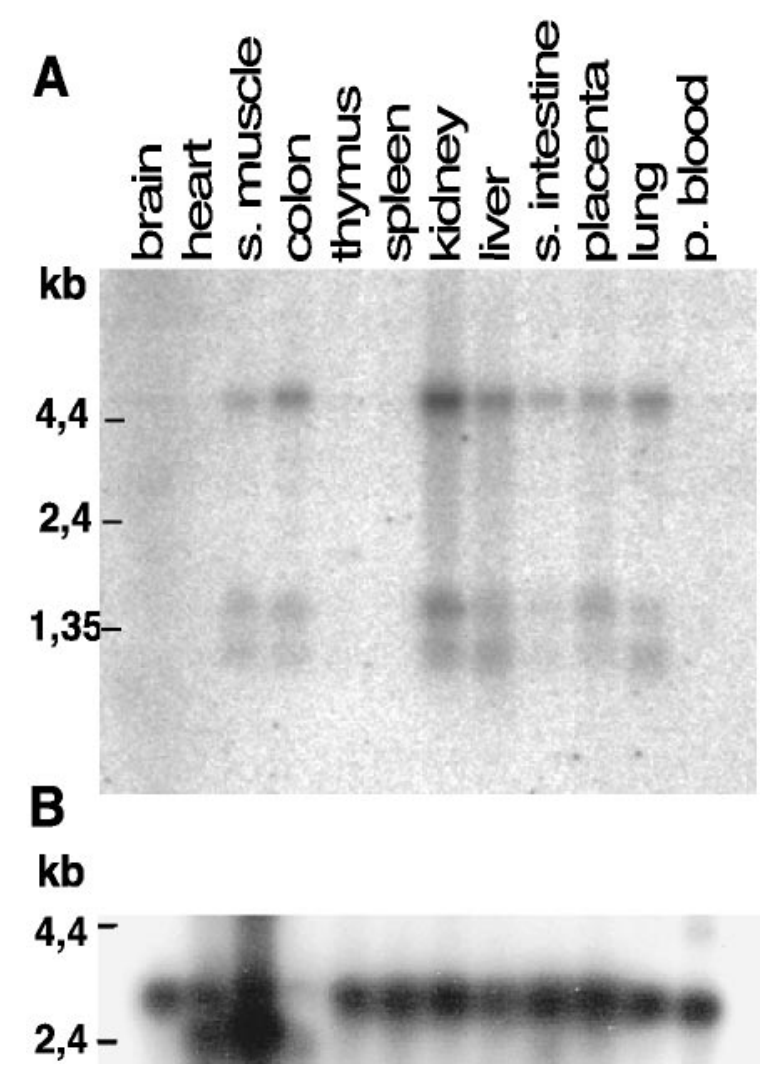

Figure 1. Northern blot on human tissues showing the expression pattern of hCRB3. (A) Multiple human tissue Northern blot from BD Biosciences Clontech was probed with a cDNA coding for hCRB3 (whole coding sequence) labeled with $\left[{ }^{32} \mathrm{P}\right] \mathrm{dCTP}$ by random priming. (B) The same membrane was stripped and reprobed with control probe for actin generated by the same method. Molecular weights in kilobases $(\mathrm{kb})$ are indicated on the left.

morphogenesis has not yet been addressed. CRB1 expression is restricted to retina and some areas of the brain, both in human and mouse (den Hollander et al., 2002) and thus cannot play a major role in the morphogenesis of epithelial tissues. So far, there is little information about CRB2 expression, but we could not detect its expression by RT-PCR in Caco-2 cells, a human epithelial cell line derived from colon (Lemmers et al., 2002). We thus looked for CRB3 mRNA expression in human tissues to evaluate its possible contribution to epithelial morphogenesis (Figure 1). CRB3 mRNAs are present in all epithelial tissues tested (colon, small intestine, liver, kidney, placenta, and lung) and also in skeletal muscle and very weakly in brain, but no signal was detected in the other tissues. Three different transcripts of 1.1, 1.4, and $4.5 \mathrm{~kb}$ were observed and they were all coexpressed in the same tissues. The 1.1- and 1.4-kb transcripts correspond to the two mRNAs found in the databases (1087 and 1443, respectively). Because we have not found in the EST databases longer isoforms of CRB3 that could account for the $4.5-\mathrm{kb}$ transcript, we looked more closely at the genomic region coding for CRB3 in the human genome. CRB3 is made up of only four exons with the last exon containing the untranslated 3' tail, perfectly fitting the two small transcripts found in EST databases. Because the genomic organization of CRB3 cannot thus give rise to such a large mRNA as 4.5 $\mathrm{kb}$, we studied the organization of the next gene (NM079174). This gene is transcribed in the opposite direction of
CRB3, and there is a possibility that it overlaps with CRB3. Given their intricate organization, it is likely that the two genes share common regulatory sequences that could also explain their common transcription in the tissues tested. Future work should help to understand this complex point.

\section{CRB3 Localizes to the Apical Membrane}

To determine the localization of CRB3 in epithelial cells, we produced antibodies against the cytoplasmic domain of hCRB3 and tested their specificity on COS-7 cells expressing either hCRB3 or constructs encoding the cytoplasmic domain of either CRB1 or CRB2 with the transmembrane domain of Crumbs and a VSV-G tag replacing the extracellular domain. By immunoblotting and immunofluorescence, we observed minimal cross-reactivity, indicating that anti-CRB3 sera were specific for CRB3 and could be used for in vivo staining (our unpublished data). On sections of mouse intestine, both sera showed a reaction with the apical membrane of enterocytes. This staining was also concentrated at the level of tight junctions and could be seen as strands in some of the epithelial cells that are in the plane of the sections (Figure 2A). By immunoelectron microscopy on frozen sections of human colon, CRB3 was found on the apical membrane and in the upper part of tight junctions (Figure 2B).

To confirm this apical localization and to look for a potential role of CRB3 in epithelial cells, we decided to use both a cDNA coding for hCRB3 and a chimera in which the extracellular domain of hCRB3 was replaced by the extracellular domain of the p75NTR, providing an efficient tag recognized by the mAb ME 20-4 (Figure 3A). hCRB3 and p75-CRB3 were expressed after transfection and selection, in MDCK cells, a renal epithelial cell line that is polarized. In these cells, both constructs, when expressed at moderate levels, were localized at the apical membrane, colocalizing with BC44 or GP114, two markers of the apical membrane (Beau et al., 1998) (Figure 3B). These data confirmed that exogenous CRB3 is an apical protein with the same subcellular distribution as CRB3 in colon.

\section{Expression of CRB3 Induced a Delay in the Establishment of Tight Junctions}

In Drosophila, overexpression of Crumbs leads in some cases to expansion of the apical domain and to disorganization of the epithelial layer (Wodarz et al., 1995). To investigate whether CRB3 could have such an effect in mammalian epithelial cells, we used MDCK transfected with hCRB3 and selected two clones with different levels of expression of hCRB3 as shown by Western blotting (Figure 4A). The polarity of hCRB3 in these clones was checked by indirect immunofluorescence after 3-4 d of confluence on Transwell filters, and both clones showed heterogeneity in expression with low- and high-expressing cells. In strongly overexpressing MDCK cells, hCRB3 was found all over the apical membrane and was also found on the upper part of the lateral membrane, showing some overlap with BC 11 (Figure $3 \mathrm{~B}$, top right). Endogenous markers such as BC44 (an apical marker), BC11 (a basolateral marker), or occludin (a marker of TJs), however, showed no mislocalization, indicating that there was no general loss of polarity in these cells (Figures $3 \mathrm{~B}$ and $4 \mathrm{~B}$ ). Because polarity is a multistep process, CRB3 could be involved in the first steps of epithelial organization as was shown in Drosophila, but its overexpression might have no dramatic effect on cells grown at confluence on filters for several days, once junctions and the general trafficking events are stabilized. To address that point, MDCK cells expressing hCRB3 or control cells were seeded at confluence in normal medium for $18 \mathrm{~h}$ and then switched to a 


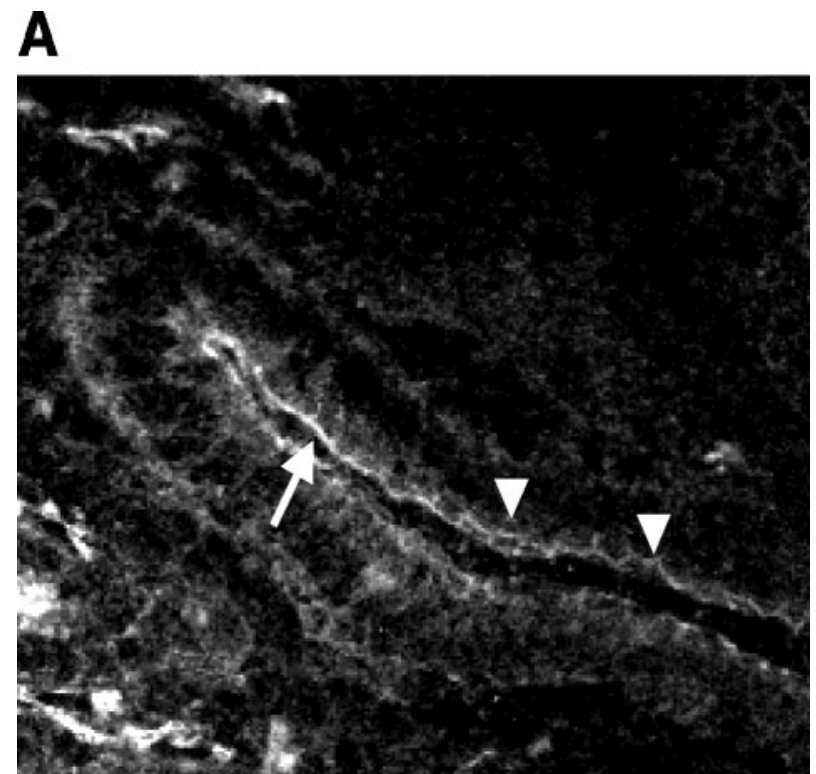

B

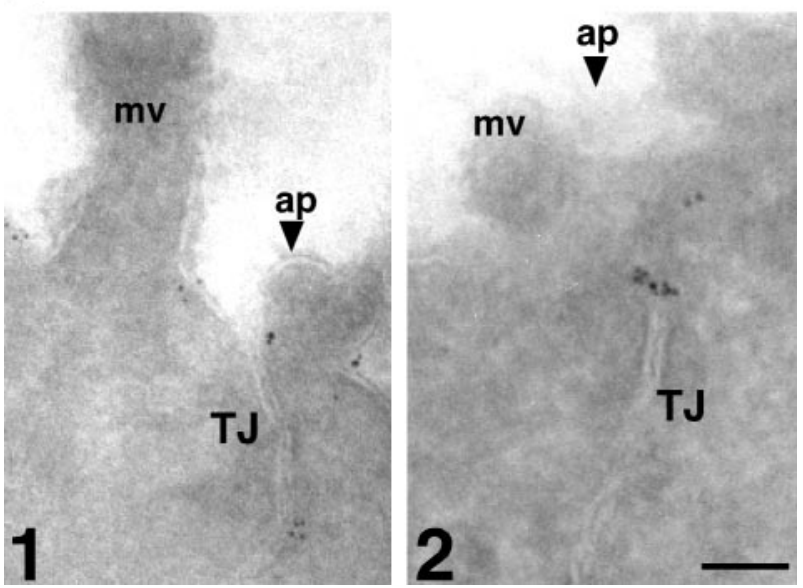

Figure 2. CRB3 localizes to the apical membrane of epithelial cells. (A) Cryosection of mouse intestine was labeled with rabbit polyclonal antibodies against CRB3. Arrow indicates the apical membrane of enterocytes, whereas arrowheads point at the apical junctions. Bar, $20 \mu \mathrm{m}$. (B) Ultrathin frozen sections of human colon were labeled with rabbit antibodies against CRB3 and revealed with secondary antibodies coupled to 6-nm gold particles. Two representative pictures are shown (1 and 2). Mv, microvilli; ap, apical membrane (marked by an arrowhead); TJ, tight junctions. Bar, $0.1 \mu \mathrm{m}$.

low calcium medium for $18 \mathrm{~h}$ to prevent formation of junctions and allowed to polarize by switching to normal medium for 3 or $6 \mathrm{~h}$. Cells were then processed for indirect immunofluorescence by using polyclonal antibodies against ZO-3 or PATJ markers of TJs (Figure 4C). MDCK cells expressing hCRB3 showed incomplete staining of both markers (Figure 4C, arrowheads) as opposed to control MDCK cells that showed intact TJs. After $6 \mathrm{~h}$, cells overexpressing hCRB3 showed, for a majority, a complete belt of TJs (our unpublished data), indicating that overexpression of hCRB3 led to a delay in the establishment of junctional complexes.
A

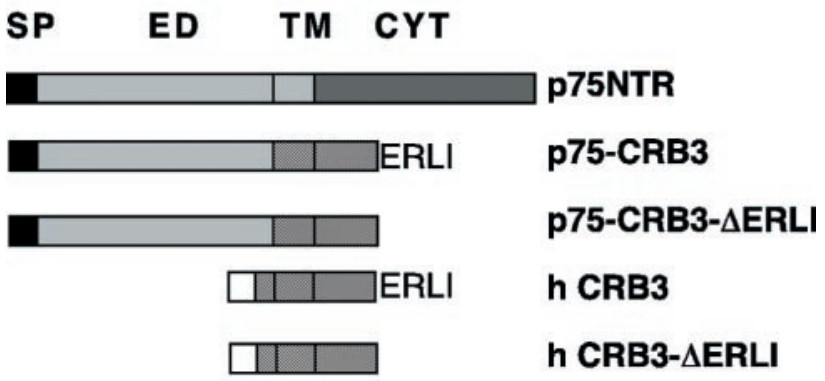

B
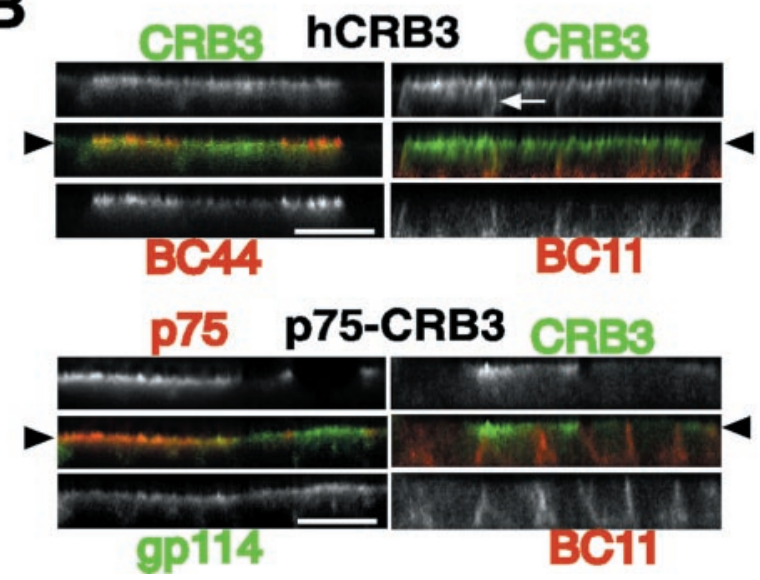

Figure 3. hCRB3 and p75-CRB3 are localized apically. (A) Schematic representation of hCRB3 constructs used in this work. SP, signal peptide of p75NTR (black boxes) or hCRB3 (empty boxes) ED, extracellular domain of p75NTR; TM, transmembrane domain of p75NTR (gray dots) or hCRB3 (black lines); CYT, cytoplasmic domain of p75NTR (gray dots) or hCRB3 (black lines); ERLI, last four amino acids of hCRB3 (deleted in p75-CRB3- $\triangle E R L I$ and in hCRB3- $\Delta$ ERLI). (B) MDCK cells expressing hCRB3 or p75-CRB3 grown at confluence for $4 \mathrm{~d}$ on Transwell filters were processed for indirect immunofluorescence. hCRB3-expressing cells were double labeled with rabbit polyclonal antibodies anti-CRB3 (in green, top) and a mouse $\mathrm{mAb}$ against an apical marker (BC44) or a basolateral marker (BC11) (in red, bottom). p75-CRB3-expressing cells were double labeled with a mouse mAb ME 20-4 against p75NTR extracellular domain (in green, top left) or rabbit polyclonal antibodies anti-CRB3 (in green, top right) and polyclonal antibodies against gp114, an apical marker (in red, bottom left) or a mouse mAb against BC11 (in red, bottom right). An arrowhead shows the level of the apical membrane, whereas arrows mark CRB3 extending along the lateral membrane. Bar, $10 \mu \mathrm{m}$.

The C-Terminal Part of CRB3 Is Involved in the Perturbation of TJs upon Overexpression

MDCK cells expressing hCRB3 offered several limitations to dissect the mechanisms involved during overexpression for several reasons. First, the heterogeneity of expression of hCRB3 made it difficult to measure general properties of the monolayer such as transepithelial resistance, tightness, or growth in tridimensional gels. Second, the antibodies currently available were raised against the cytoplasmic domain of CRB3 and do not react well (or at all) with the deleted form $\triangle$ ERLI (our unpublished data), preventing the use of this loss of function mutant as was done in Drosophila (Klebes and Knust, 2000). To circumvent that problem, we 


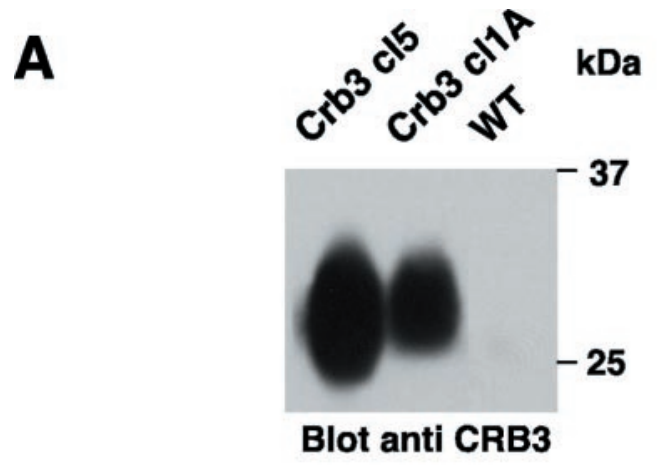

B

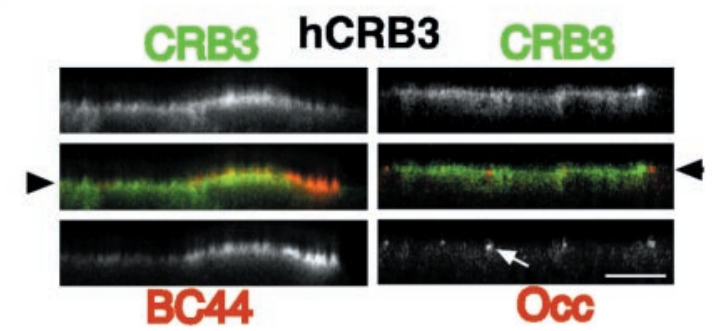

C

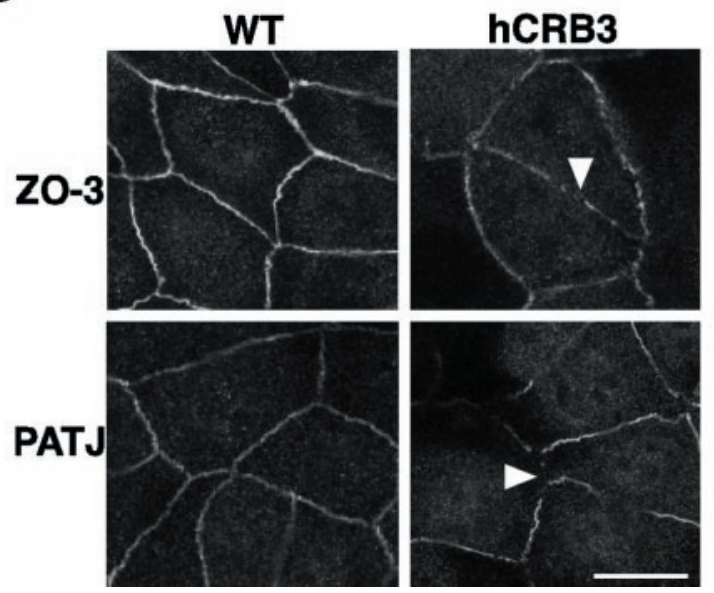

Figure 4. Overexpression of hCRB3 in MDCK cells. (A) Identical amounts of membrane proteins from two clones of MDCK cells expressing hCRB3 (CRB3 $\mathrm{cl} 5$ and 1A) were processed together with untransfected MDCK (WT) for SDS-PAGE analysis and Western blotting. CRB3 was detected using polyclonal antibodies against its cytoplasmic domain. Molecular mass marker ( 25 and $37 \mathrm{kDa}$ ) migration is shown on the right. (B) MDCK cells expressing hCRB3 and grown at confluence for $4 \mathrm{~d}$ on Transwell filters were processed for indirect immunofluorescence. hCRB3-expressing cells were double labeled with rabbit polyclonal antibodies anti-CRB3 (in green, top) and a mouse mAb against an apical marker (BC44) or a tight junction marker, occludin (Occ) (in red, bottom left and right, respectively). An arrowhead shows the level of the apical membrane, whereas arrows mark the position of TJs. Bar, $10 \mu \mathrm{m}$. (C) Control MDCK cells (WT) or MDCK cells expressing hCRB3 (hCRB3) were seeded at confluence onto Transwell filters and kept in a low calcium medium for overnight to prevent the formation of TJs. At time 0 , cells were switched to normal medium for $3 \mathrm{~h}$ and processed for indirect immunofluorescence with rabbit polyclonal antibodies against two TJ markers, ZO-3 and PATJ. Arrowheads point at discontinuities in TJ integrity. Bar, $10 \mu \mathrm{m}$. used the p75-CRB3 and p75-CRB3 EERLI chimeras. MDCK cells stably transfected with these two constructs were cloned and used for further studies of the effect of overexpression upon epithelial organization. For each construct, a mixed population of positive clones and two independent clones were selected and studied for possible changes in cell morphology and polarity. Apical localization was not dependent on the presence of the ERLI because p75CRB3 $\triangle$ ERLI was also enriched on the apical side of MDCK cells (Figure 5A). To ascertain that p75-CRB3 was also engaged in supramolecular complexes, we tested its association with PATJ by coimmunoprecipitation from MDCK cell lysates. As expected, neither p75-CRB3 $\triangle$ ERLI nor p75NTR was able to pull down endogenous PATJ, whereas p75-CRB3 did (our unpublished data), indicating that p75-CRB3 could be used as a model to study the role of the intracellular and transmembrane domain of CRB3. When cells expressing p75-CRB3 were grown to confluence on Transwell filters, there was no obvious modification of cell polarity as was found for hCRB3, indicating that p75-CRB3 did not induce more perturbations than its untagged counterpart (Figures $3 \mathrm{~B}$ and $4 \mathrm{~B}$ ). It has been reported, however, that when MDCK cells are grown in tridimensional gels, they exhibit phenotypes that are not detectable in monolayer culture conditions (O'Brien et al., 2002). For each construct, positive clones were grown in $60 \%$ Matrigel for 7-10 d to form cysts and then double-labeled with the mouse $\mathrm{mAb}$ against the extracellular domain of p75NTR (ME 20-4) and rabbit antibodies against ZO-1, a protein of the tight junctions. Under these culture conditions, a strong effect on the organization of the epithelial layer was observed in MDCK cells expressing p75-CRB3. Control MDCK cells expressing p75NTR made cysts with a round-shaped lumen and well ordered tight junctions (Figure 5A). In contrast, p75-CRB3-expressing cells showed a very irregular apical membrane with deep invaginations into the monolayer, sometimes almost reaching the basal side of the cyst (see arrows in Figure 5A, middle). This disorganization of the lumen of the cysts was observed in most of them $(>90 \%)$, and it was not observed with MDCK cells expressing p75-CRB3 $\triangle E R L I$, indicating that this effect was mediated by potential interactions with PDZ containing proteins. Apical/basal polarity was not affected, however, because both p75-CRB3 and p75-CRB3 $\triangle$ ERLI were only accumulated on the apical side (Figure $5 \mathrm{~A}$ ), whereas BC11, a basolateral marker, was not depolarized either (our unpublished data). To confirm the disorganization of the lumen of the cysts formed by MDCK cells expressing p75-CRB3, we performed electron microscopy on these cells grown in Matrigel for $10 \mathrm{~d}$. In p75-CRB3 cysts, deep invaginations of the apical membrane covered with microvilli were observed, whereas it was not the case in p75-CRB3 $\triangle E R L I$ cysts. At this level of resolution, however, we could not detect any dramatic morphological change in the tight junctions (Figure 5B).

The morphology of p75-CRB3 cells in cysts, compared with control MDCK cells, prompted us to test whether p75CRB3 expression had an effect on junction assembly/disassembly and tightness. For that purpose, parental MDCK cells and two clones of p75-CRB3 and p75-CRB3 $\Delta$ ERLI were seeded at the same high density on Transwell filters, and formation of functional tight junctions was followed by addition of $\left[{ }^{3} \mathrm{H}\right]$ inulin in the apical chamber after 12 and $24 \mathrm{~h}$ of culture. The amount of $\left[{ }^{3} \mathrm{H}\right]$ inulin found in the basal chamber was measured after $1 \mathrm{~h}$ and expressed as a percentage of the total radioactivity (Figure 6A). Control MDCK monolayers were tight already after $12 \mathrm{~h}$ with $<1 \%$ of leakage. In contrast, p75-CRB3 monolayers showed levels of 


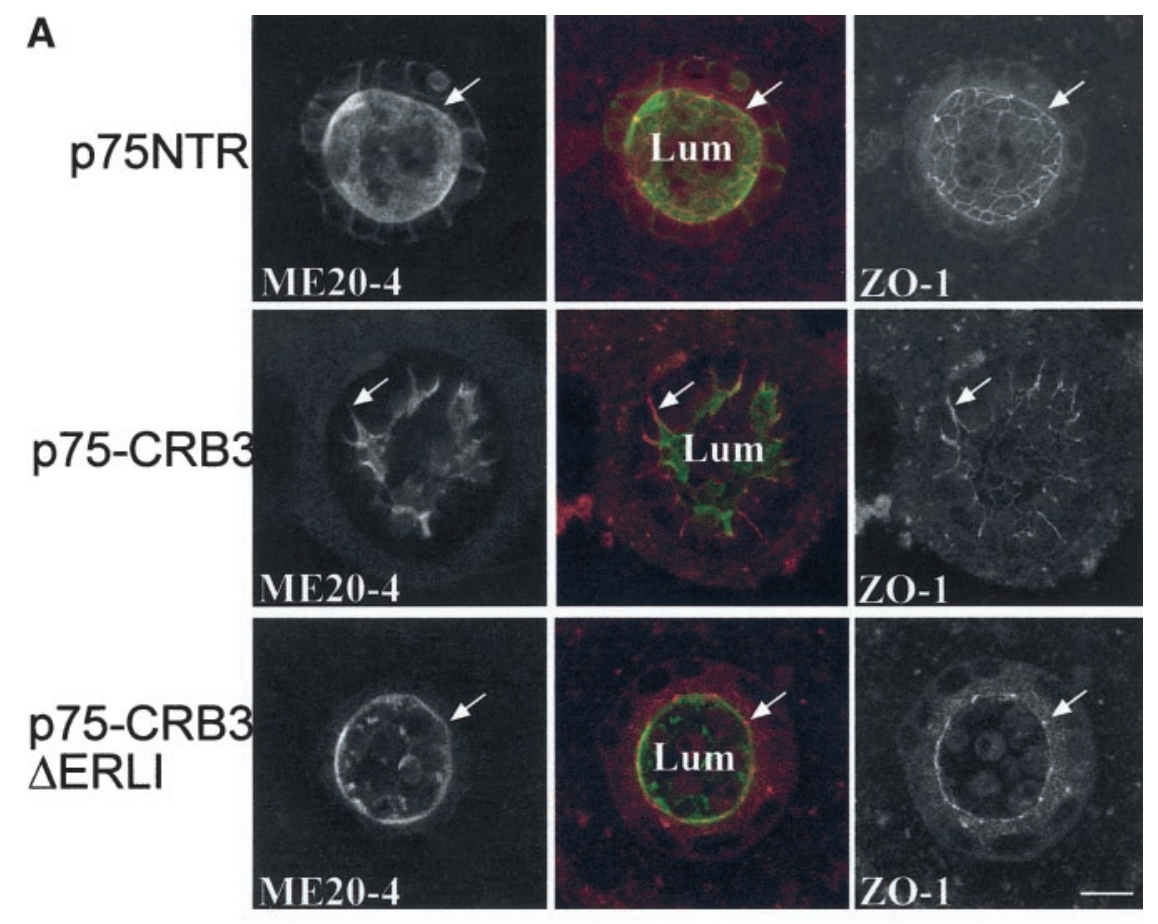

\section{B}

Figure 5. Expression of p75-CRB3 induces a change in the morphology of the apical membrane. (A) MDCK cells expressing either p75NTR, p75-CRB3, or p75-CRB3- $\Delta$ ERLI were grown in Matrigel gels for $7-10 \mathrm{~d}$ and processed for indirect immunofluorescence and double labeled with a mouse mAb ME 20-4 against p75NTR extracellular domain (in green) and a polyclonal antibodies against ZO-1 (in red). Confocal sections passing though the middle of each cyst are shown. Arrows point at tight junctions. Lum, lumen of the cyst with some dying cells in it. Bar, $10 \mu \mathrm{m}$. (B) Electron micrographs showing the organization of the cells in cysts expressing either p75-CRB3 or p75-CRB3- $\Delta$ ERLI. The apical membrane (ap) is covered with numerous microvilli (mv), whereas the basal membrane (b) is contacting the Matrigel (asterisk). D, desmosomes. Picture 1 and 2 are magnified 6300 and 6000 times, whereas 3 and 4 are magnified 33,000 and 60,000 times, respectively. In picture 1 , the apical membrane from the lumen of the cyst is deeply invaginated between adjacent cells explaining the ZO-1 labeling observed in A.
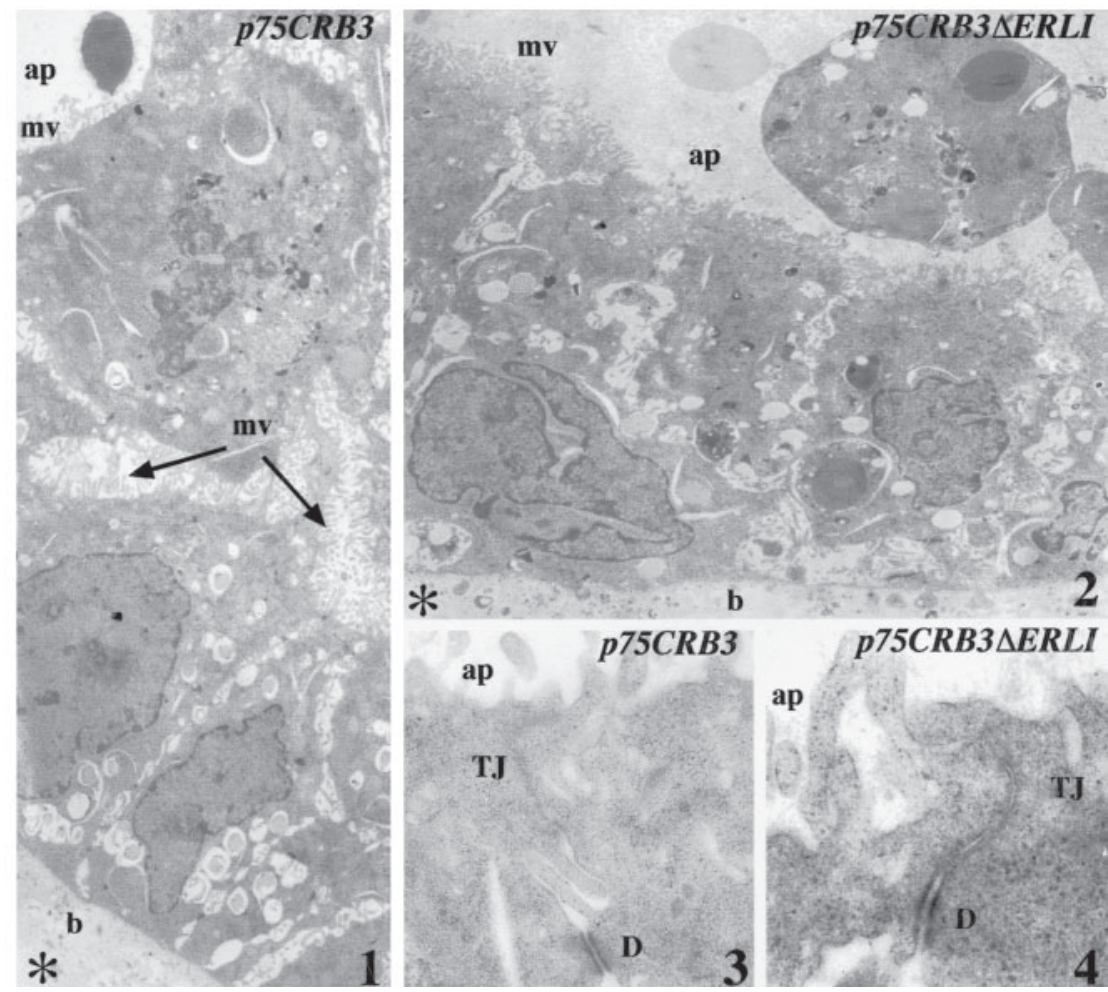

leakage that were 7-14 times higher. Even after $24 \mathrm{~h}$ of culture, these levels were still 4-8 times higher than control levels, indicating that tight junctions were not fully functional. This effect of p75-CRB3 expression on the monolayer was not produced by the expression of p75-CRB3 $\Delta$ ERLI, pointing again at the potential role of a protein complex binding to the last amino acids of CRB3. We next tested the stability of tight junctions in confluent monolayers of MDCK cells expressing p75-CRB3, p75-CRB3 $\triangle$ ERLI, and parental cells by washing the cells with phosphate-buffered saline containing $2 \mathrm{mM}$ EGTA and keeping them with culture medium without calcium. Cells were then fixed and treated 


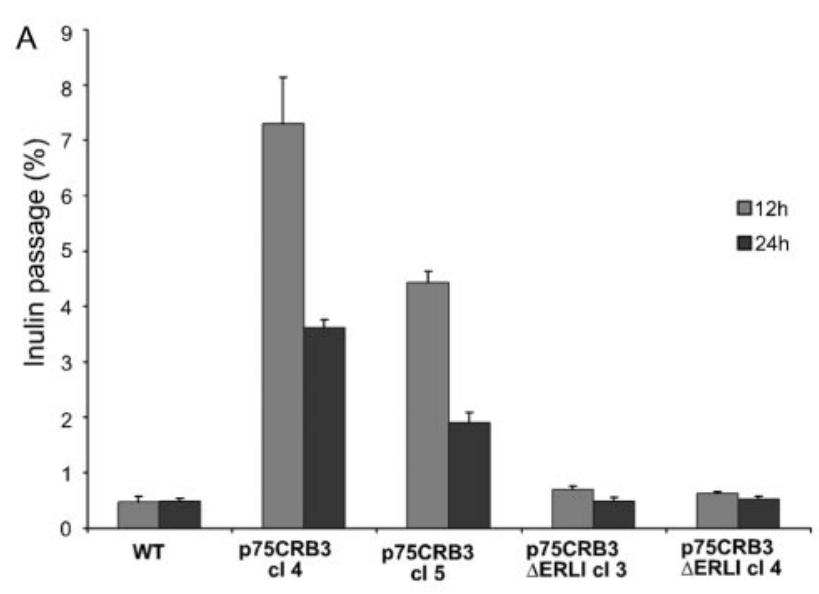

B

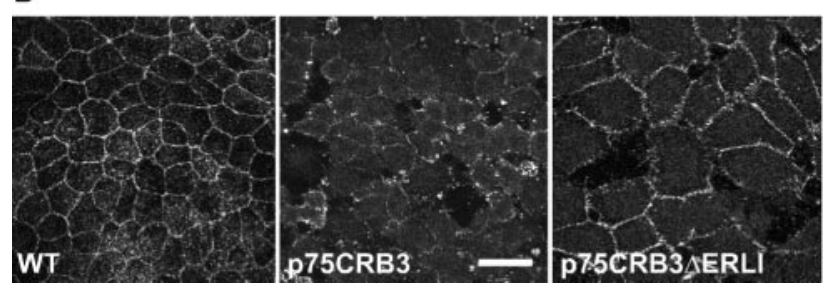

Figure 6. Overexpression of p75-CRB3 regulates negatively tight junctions in MDCK cells. (A) Untransfected MDCK cells (WT) and two clones of MDCK cells expressing either p75-CRB3 (cl4 and cl5) or p75-CRB3- $\Delta$ ERLI (cl3 and cl4) were seeded at confluence on Transwell filters and tested for inulin passage in $1 \mathrm{~h}$ from the apical to the basolateral chambers after $12 \mathrm{~h}$ (gray boxes) and $24 \mathrm{~h}$ (dark boxes) of culture in normal conditions. Inulin passage is expressed as the percentage of $\left[{ }^{3} \mathrm{H}\right]$ inulin $( \pm \mathrm{SD})$ found in the basolateral chamber over the total amount of inulin applied $(n=4)$. (B) Untransfected MDCK cells (WT) and MDCK cells expressing either p75-CRB3 or p75-CRB3- $\triangle$ ERLI were grown at confluence for $3 \mathrm{~d}$ and then submitted to calcium deprivation to open tight junctions. Cells were then fixed and processed for indirect immunofluorescence with antibodies against occludin, a marker of the zonula occludens. Bar, $10 \mu \mathrm{m}$.

for immunofluorescence by using occludin as a marker of tight junctions. MDCK cells expressing p75-CRB3 had lost occludin labeling already after $10 \mathrm{~min}$ without calcium into the culture medium, whereas control and p75-CRB3 $\Delta$ ERLI cells still had peripheral staining of occludin (Figure 6B). Thus, expression of p75-CRB3 led to less stable junctions, confirming the data obtained with overexpressed hCRB3 and its potential role in normal tight junction building and maintenance.

\section{The C-Terminal Part of CRB3 Interacts Directly with Par6 PDZ Domain}

Because the effect of overexpression of hCRB3 or p75-CRB3 on TJs establishment and stability relied on the presence of the ERLI motif, it should be mediated by interactions between this domain of CRB3 and protein complexes involved in TJ regulation. Among the known partners of CRB3, only Pals1 (the homologue of Sdt) binds directly to the amino acids ERLI of CRB3 (Roh et al., 2002b), but so far there is no documented role (either by loss or gain of function) for Pals1 in epithelial polarity and TJ assembly. Thus, we performed a two-hybrid screen by using the cytoplasmic domain of CRB3 as bait and a cDNA library made from human breast

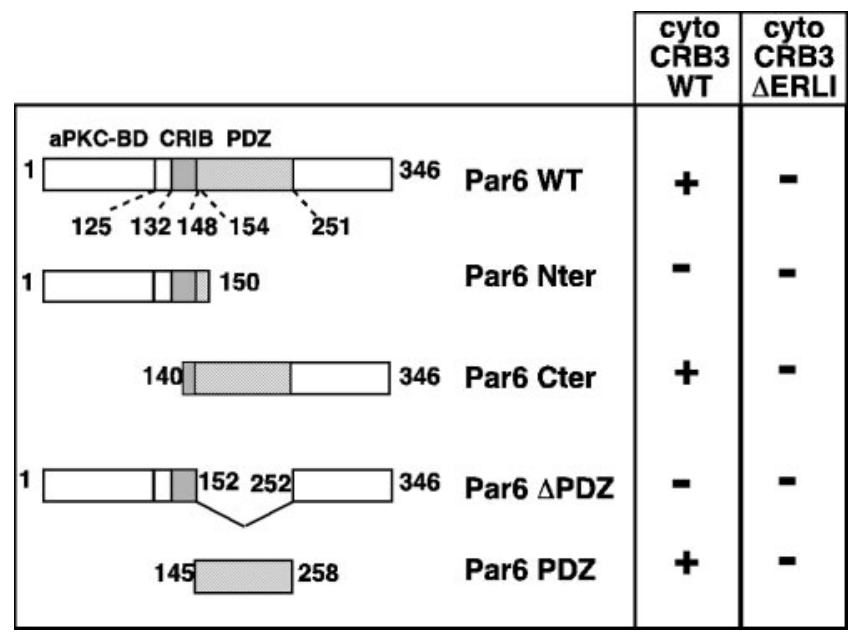

Figure 7. hCRB3 interacts with hPar6 $\alpha$ in two-hybrid assays. Several constructs encoding different parts of Par6 $\alpha$ (Par6 WT) were designed and tested for their interaction with the cytoplasmic domain of hCRB3 (cyto CRB3). Par6 Nter, amino acids from 1-150 (encompassing the semi-CRIB domain in gray dots). Par6 Cter, amino acids from 140 to 346, including the PDZ domain (hatched bars). Par6 $\triangle \mathrm{PDZ}$, full-length Par6 $\alpha$ deleted of the amino acids 152-252 comprising the PDZ domain. Par6 PDZ, amino acids from 145 to 258 corresponding to the functional PDZ domain. Par6 WT, Cter, and PDZ all interacted (+) with cyto CRB3 WT but not with $\Delta$ ERLI (-).

tissue. One positive cDNA represented $75 \%$ of all clones and after sequencing was found identical to hPar $6 \alpha$. We used the two-hybrid assay to identify the molecular determinants involved in the interaction between CRB3 and Par6. The binding between Par6 $\alpha$ and CRB3 relied on the presence of the ERLI residues in CRB3 and of the PDZ domain of Par $6 \alpha$ because deletion of either of these domains abolished growth in selective medium and activation of the $\beta$-galactosidase (Figure 7). To confirm that the binding between CRB3 and Par6 $\alpha$ was direct, recombinant Par6PDZ fused to GST was used as a bait to trap increasing amounts of purified His-CRB3 cytoplasmic domain (His-CRB3cyt) (Figure 8A). Binding of His-CRB3cyt on GST-Par6 PDZ beads was dose dependent and saturable and exhibited an apparent affinity of $\sim 300 \mathrm{nM}$ (Figure 8A; our unpublished data). We then tested whether Par6 $\alpha$ could be purified by its affinity for a GST-CRB3cyt fusion protein by using a lysate of COS-7 cells transfected with Par6 $\alpha$ or with only the C-terminal part of Par6 $\alpha$ containing its PDZ domain (Par6 C-ter). As expected, the GST-CRB3cyt fusion protein but not the GSTCRB3 $\triangle$ ERLI brought down Par6 $\alpha$ and Par6 C-ter (Figure 8B). Finally, to demonstrate that a complex between CRB3 and Par6 $\alpha$ was present in epithelial cells, we performed coimmunoprecipitations. The use of MDCK cells expressing p75NTR, p75-CRB3, or p75-CRB3- $\Delta$ ERLI and of ME 20.4 antibodies allowed us to immunoprecipitate CRB3 without interfering with any interactions occurring with its cytoplasmic domain (Figure 8C). Endogenous Par6 was only detected in the immunoprecipitates from p75-CRB3-expressing cells, confirming that the interaction between CRB3 and Par6 occurred in epithelial cells in culture and that it also relied on the presence of the ERLI residues.

\section{DISCUSSION}

Crumbs belongs to a class of proteins involved in the organization of the plasma membrane into specialized domains, 
A

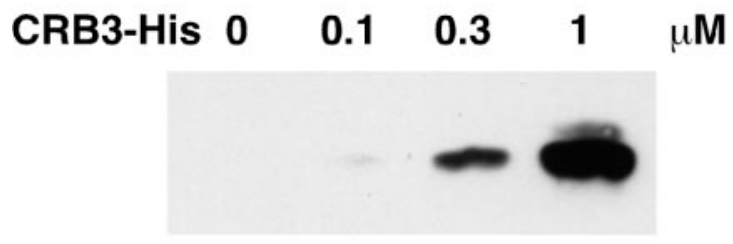

B

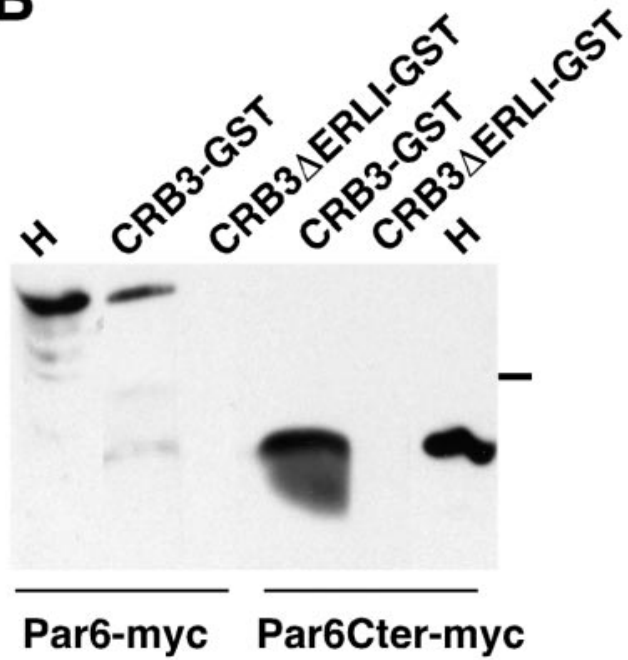

C

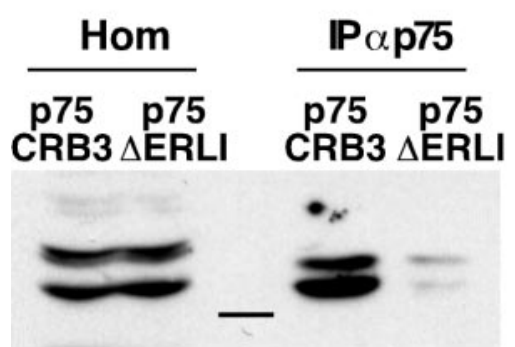

Blot anti-Par6

Figure 8. hCRB3 interacts with hPar6 $\alpha$ in vitro and in vivo. (A) Soluble recombinant cytoplasmic domain of hCRB3 tagged with six histidine residues (CRB3) $(0-3 \mu \mathrm{g})$ was incubated in increasing concentrations with an excess of the PDZ domain of hPar6 $\alpha$ coupled to GST and coated on glutathione-Sepharose beads. After washing, the beads were eluted and the bound CRB3-His was analyzed by SDS-PAGE and detected by Western blotting with polyclonal antibodies against CRB3. (B) The cytoplasmic domain of hCRB3 (with or without the ERLI amino acids) fused to GST was coated on glutathione-Sepharose beads (CRB3-GST and CRB3AERLIGST, respectively). These beads were incubated with lysates $(200 \mu \mathrm{g})$ from COS-7 cells transfected either with Par6 $\alpha$ tagged with the myc epitope (Par6-myc) or with the C-terminal half of Par6, including the PDZ domain (Par6Cter-myc). After washing, the beads were eluted and the bound Par6 was analyzed by Western blotting with a mAb against myc. $\mathrm{H}$, homogenates $(50 \mu \mathrm{g})$ from COS-7 cells transfected either with Par6 $\alpha$ or the C-ter of Par6 $\alpha$ tagged with the myc epitope. Molecular mass marker $(37 \mathrm{kDa})$ migration is indicated between the two panels. (C) MDCK cells expressing either p75-CRB3 or p75-CRB3 $\triangle$ ERLI were lysed and processed for immunoprecipitation with mouse ME 20-4 as described in MATERIALS AND METHODS. Par6 contained in homogenates (Hom) and immunoprecipitates (IP $\alpha$ p75) was detected using rabbit polyclonal antibodies. Molecular mass marker $(37 \mathrm{kDa})$ migration is shown in the center. which is a hallmark of epithelial cells throughout the animal kingdom. In Drosophila, Crumbs is involved both in the development of the ectodermal layer during gastrulation (Tepass et al., 1990) and in the development of eye photoreceptors during larval stages (Izaddoost et al., 2002; Johnston and Gallant, 2002; Pellikka et al., 2002). It is thus of importance to investigate whether such a gene and such a function are conserved in mammals. Thus, if a crucial function of Crumbs in epithelial polarity and morphogenesis was conserved, there must be at least another Crumbs expressed by epithelial cells in mammals. CRB1 was the first human homologue of Crumbs to be described, but its expression pattern is restricted to eye and brain in mouse and human (den Hollander et al., 1999; den Hollander et al., 2002). In addition, two other proteins with a highly conserved cytoplasmic domain can be found in the databases (Lemmers et al., 2002; Roh et al., 2002b). These two proteins called CRB2 and 3 differ in their extracellular domain because CRB2 has a similar organization to Crumbs and CRB1, whereas CRB3 lacks the EGF and laminin-like repeats (Medina et al., 2002a). We do not favor CRB2 as a general epithelial Crumbs, however, because only CRB3 was detected by RT-PCR on Caco-2, a human epithelial cell line (Lemmers et al., 2002). In contrast, we have several arguments to think that CRB3 is a genuine epithelial Crumbs despite is short extracellular domain. First, CRB3 shows a highly conserved cytoplasmic domain with the two signature motifs GTY and ERLI that are important for the epithelial function of Crumbs in ectodermal cells (Klebes and Knust, 2000). In particular, it has been shown that the ERLI motif binds to Stardust, which in turn recruits Discs lost (Bachmann et al., 2001; Hong et al., 2001; Roh et al., 2002b), whereas we showed recently that the GTY region is involved in recruiting a DMoesin and $\beta$-Heavy-spectrin network in Drosophila ectodermal cells (Medina et al., 2002b). Furthermore, we and others showed recently that CRB3 is able to coimmunoprecipitate with PATJ, the human homologue of Discs lost, confirming that both are parts of a cellular complex in epithelial cells (Lemmers et al., 2002; Roh et al., 2002a,b). Also, the localization and effects of CRB3 in epithelial cells described in this study are very similar to what was observed in Drosophila upon overexpression of a Crumbs construct in which the myc epitope replaces the extracellular domain (Wodarz et al., 1995). CRB3 is present on the apical membrane of epithelial intestinal cells and is accumulated at the apex of TJs, a localization reminiscent of Crumbs distribution in ectodermal cells (Tepass et al., 1990) and in fact, a recent study confirmed this apical localization of endogenous CRB3 in MDCK cells (Makarova et al., 2003). In Drosophila, overexpression of Crumbs led to a loss of the ZAs and to a multilayered epithelium (Wodarz et al., 1995), whereas in MDCK cells there is no disruption of occludin distribution and no gross loss of polarity at steady state, but we could observe a delay in the formation of an intact belt of TJs. Thus, both structural and functional data on CRB3 indicate that it is likely a member of the Crumbs family.

To further analyze CRB3 overexpression phenotype in epithelial cells, a chimera between the extracellular domain of p75NTR and the transmembrane and cytoplasmic domains of CRB3 was stably transfected in MDCK cells. p75CRB3 chimera localized at the apical membrane like hCRB3 and was able to coprecipitate PATJ, indicating that it was behaving like CRB3 (Lemmers et al., 2002; Makarova et al., 2003). As for hCRB3-overexpressing MDCK cells, we did not observe a dramatic loss of polarity in MDCK cells overexpressing $\mathrm{p} 75-\mathrm{CRB} 3$, indicating that in mammals several mechanisms can compensate a defective pathway for the 
building of junctions and cell polarity. Indeed, the presence of transmembrane proteins such as occludin and claudins in the TJs of mammalian epithelial cells, could compensate to some extend the effects of CRB3 overexpression. A clear functional defect in the building and stability of TJs was observed; however, in agreement with the data obtained in a recent study by using a myc-tagged hCRB3 (Roh et al., 2003). In contrast with data reported by Roh and colleagues, we observed that even in strongly overexpressing cells, two endogenous apical markers were still restricted to the apical membrane, whereas hCRB3 was delocalized to the lateral membrane. Loss of function of CRB3 will probably help to clarify the exact role of CRB3 in epithelial polarity acquisition or maintenance. MDCK cells are from canine origin, however, and the sequence of dog CRB3 is not known yet, precluding the use of RNA interference in these cells. Despite these drawbacks the loss of function approach will have to be developed in the near future to confirm our data.

In Drosophila, Crumbs combines two functions, one relying on the cytoplasmic domain and another on the extracellular domain. The function of the cytoplasmic domain is to allow the correct assembly and positioning of the ZAs, thus determining the borders of the apical membrane (Knust, 2000), whereas the extracellular domain is involved in the elongation of the stalk membrane of photoreceptors (Izaddoost et al., 2002; Johnston and Gallant, 2002; Pellikka et al., 2002). In mammals, CRB3, which is present in most epithelial cells, might have lost the function of the extracellular domain not relevant to polarity and junction formation, whereas CRB1 has kept both functions in the retina. Our hypothesis is that CRB3 regulates TJ formation through its cytoplasmic domain and interactions with several cytosolic partners. Here, we showed that CRB3 binds directly to Par6, which is a well-known regulator of tight junction formation together with Par3 and atypical protein kinase C (Ohno, 2001). It has been shown that overexpression of Par6 in MDCK cells leads to a delay in the assembly of functional TJs (Gao et al., 2002), a phenotype very similar to what we observed for CRB3 overexpression. Thus, we propose that the two proteins are in the same cellular pathway for the building of TJs and act in a similar manner. In addition, it is known that Pals1, the homologue of Stardust, binds directly to the last amino acids ERLI of CRB3 (Roh et al., 2002b). There is, however, no documented cellular role for Pals1 so far, but a recent report has shown that Pals1 can bind to Par6 (Hurd et al., 2003). Thus, in addition to the direct binding we demonstrated in this work, an indirect connection between CRB3 and Par6 might regulate in vivo the formation and stability of tight junctions. PATJ, the homologue of Dlt, is likely to be also a major player in this pathway because we showed that its overexpression in MDCK cells disrupts ZO-3 labeling (Lemmers et al., 2002), and it has been reported that both proteins can interact in vitro (Roh et al., 2002a). A very recent study in Drosophila has shown that there is a direct binding between dPar6 and Dlt, increasing even further the intricacy of the Crumbs molecular complex (Nam and Choi, 2003). A better understanding of the molecular network linking the CRB3 complex to Par6 during cell polarization and TJ formation might help to shed light on these particularly important steps in cell polarity and epithelial morphogenesis.

\section{ACKNOWLEDGMENTS}

We thank colleagues from the Crumbs consortium who shared information and reagents used in this study and for critical comments on this manuscript. We thank C. Faivre-Sarrailh and Julien Falk for helpful discussions and comments during this study and on the manuscript. This work was supported by Centre National de la Recherche Scientifique 6156, Université de la Méditerranée, Institut de Biologie du Développement de Marseille, Fondation de France and Association pour la Recherche sur le Cancer 9297, and an EC grant (Crumbs therapeutics) to A.L. C.L. is a recipient of a fellowship from the French Ministry of Education, and E.M. is a recipient of an Association pour la Recherche sur le Cancer fellowship.

\section{REFERENCES}

Bachmann, A., Schneider, M., Theilenberg, E., Grawe, F., and Knust, E. (2001) Drosophila Stardust is a partner of Crumbs in the control of epithelial cell polarity. Nature 414, 638-643.

Beau, I., Groyer-Picard, M.T., Le Bivic, A., Vannier, B., Loosfelt, H., Milgrom, E., and Misrahi, M. (1998). The basolateral localization signal of the folliclestimulating hormone receptor. J. Biol. Chem. 273, 18610-18616.

Bhat, M.A., Izaddoost, S., Lu, Y., Cho, K.O., Choi, K.W., and Bellen, H.J. (1999). Discs Lost, a novel multi-PDZ domain protein, establishes and maintains epithelial polarity. Cell 96, 833-845.

Bilder, D., Schober, M., and Perrimon, N. (2003). Integrated activity of PDZ protein complexes regulates epithelial polarity. Nat. Cell Biol. 5, 53-58.

Delgrossi, M.H., Breuza, L., Mirre, C., Chavrier, P., and Le Bivic, A. (1997). Human syntaxin 3 is localized apically in human intestinal cells. J. Cell Sci. $110,2207-2214$

den Hollander, A.I., Ghiani, M., de Kok, Y.J., Wijnholds, J., Ballabio, A., Cremers, F.P., and Broccoli, V. (2002). Isolation of Crb1, a mouse homologue of Drosophila crumbs, and analysis of its expression pattern in eye and brain. Mech. Dev. 110, 203-207.

den Hollander, et al. (2001). Leber congenital amaurosis and retinitis pigmentosa with Coats-like exudative vasculopathy are associated with mutations in the crumbs homologue 1 (CRB1) gene. Am. J. Hum. Genet. 69, 198-203.

den Hollander, et al. (1999). Mutations in a human homologue of Drosophila crumbs cause retinitis pigmentosa (RP12). Nat. Genet. 23, 217-221.

Gao, L., Joberty, G., and Macara, I.G. (2002). Assembly of epithelial tight junctions is negatively regulated by Par6. Curr. Biol. 12, 221-225.

Grawe, F., Wodarz, A., Lee, B., Knust, E., and Skaer, H. (1996). The Drosophila genes crumbs and stardust are involved in the biogenesis of adherens junctions. Development 122, 951-959.

Hong, Y., Stronach, B., Perrimon, N., Jan, L.Y., and Jan, Y.N. (2001). Drosophila Stardust interacts with Crumbs to control polarity of epithelia but not neuroblasts. Nature 414, 634-638.

Hurd, T.W., Gao, L., Roh, M.H., Macara, I.G., and Margolis, B. (2003). Direct interaction of two polarity complexes implicated in epithelial tight junction assembly. Nat. Cell Biol. 5, 137-142.

Izaddoost, S., Nam, S.C., Bhat, M.A., Bellen, H.J., and Choi, K.W. (2002) Drosophila Crumbs is a positional cue in photoreceptor adherens junctions and rhabdomeres. Nature 416, 178-183.

Johnston, L.A., and Gallant, P. (2002). Control of growth and organ size in Drosophila. Bioessays 24, 54-64.

Klebes, A., and Knust, E. (2000). A conserved motif in Crumbs is required for E-cadherin localisation and zonula adherens formation in Drosophila. Curr. Biol. 10, 76-85.

Knust, E. (2000). Control of epithelial cell shape and polarity. Curr. Opin. Genet. Dev. 10, 471-475.

Le Bivic, A., Hirn, M., and Reggio, H. (1988). HT-29 cells are an in vitro model for the generation of cell polarity in epithelia during embryonic differentiation. Proc. Natl. Acad. Sci. USA 85, 136-140.

Le Bivic, A., Sambuy, Y., Mostov, K., and Rodriguez-Boulan, E. (1990). Vectorial targeting of an endogenous apical membrane sialoglycoprotein and uvomorulin in MDCK cells. J. Cell Biol. 110, 1533-1539.

Le Bivic, A., Sambuy, Y., Patzak, A., Patil, N., Chao, M., and RodriguezBoulan, E. (1991). An internal deletion in the cytoplasmic tail reverses the apical localization of human NGF receptor in transfected MDCK cells. J. Cell Biol. 115, 607-618.

Lemmers, C., Medina, E., Delgrossi, M.H., Michel, D., Arsanto, J.P., and Le Bivic, A. (2002). hINADl/PATJ, a homolog of discs lost, interacts with crumbs and localizes to tight junctions in human epithelial cells. J. Biol. Chem. 277 $25408-25415$.

Lipschutz, J.H., Guo, W., O’Brien, L.E., Nguyen, Y.H., Novick, P., and Mostov, K.E. (2000). Exocyst is involved in cystogenesis and tubulogenesis and acts by modulating synthesis and delivery of basolateral plasma membrane and secretory proteins. Mol. Biol. Cell 11, 4259-4275. 
Makarova, O., Roh, M.H., Liu, C.J., Laurinec, S., and Margolis, B. (2003). Mammalian Crumbs3 is a small transmembrane protein linked to protein associated with Lin-7 (Pals1). Gene 302, 21-29.

Medina, E., Lemmers, C., Lane-Guermonprez, L., and Le Bivic, A. (2002a). Role of the Crumbs complex in the regulation of junction formation in Drosophila and mammalian epithelial cells. Biol. Cell 94, 305-313.

Medina, E., Williams, J., Klipfell, E., Zarnescu, D., Thomas, G., and Le Bivic, A. (2002b). Crumbs interacts with moesin and beta(Heavy)-spectrin in the apical membrane skeleton of Drosophila. J. Cell Biol. 158, 941-951.

Mostov, K.E., Verges, M., and Altschuler, Y. (2000). Membrane traffic in polarized epithelial cells. Curr. Opin. Cell Biol. 12, 483-490.

Muller, H.A. (2000). Genetic control of epithelial cell polarity: lessons from Drosophila. Dev. Dyn. 218, 52-67.

Nam, S.C., and Choi, K.W. (2003). Interaction of Par-6 and Crumbs complexes is essential for photoreceptor morphogenesis in Drosophila. Development 130, 4363-4372.

Nelson, W.J., and Yeaman, C. (2001). Protein trafficking in the exocytic pathway of polarized epithelial cells. Trends Cell Biol. 11, 483-486.

O'Brien, L.E., Zegers, M.M., and Mostov, K.E. (2002). Opinion: building epithelial architecture: insights from three-dimensional culture models. Nat. Rev. Mol. Cell. Biol. 3, 531-537.

Ohno, S. (2001). Intercellular junctions and cellular polarity: the PAR-aPKC complex, a conserved core cassette playing fundamental roles in cell polarity. Curr. Opin. Cell Biol. 13, 641-648.

Pellikka, M., Tanentzapf, G., Pinto, M., Smith, C., McGlade, C.J., Ready, D.F., and Tepass, U. (2002). Crumbs, the Drosophila homologue of human CRB1/ $\mathrm{RP} 12$, is essential for photoreceptor morphogenesis. Nature 416, 143-149.
Rashbass, P., and Skaer, H. (2000). Cell polarity: nailing Crumbs to the scaffold. Curr. Biol. 10, R234-236.

Roh, M.H., Fan, S., Liu, C.J., and Margolis, B. (2003). The Crumbs3-Pals1 complex participates in the establishment of polarity in mammalian epithelial cells. J. Cell Sci. 116, 2895-2906.

Roh, M.H., Liu, C.J., Laurinec, S., and Margolis, B. (2002a). The carboxyterminus of zona Occludens- 3 binds and recruits a mammalian homologue of discs lost to tight junctions. J. Biol. Chem. 20, 20

Roh, M.H., Makarova, O., Liu, C.J., Shin, K., Lee, S., Laurinec, S., Goyal, M., Wiggins, R., and Margolis, B. (2002b). The Maguk protein, Pals1, functions as an adapter, linking mammalian homologues of Crumbs and Discs Lost. J. Cell Biol. 157, 161-172.

Saito, H., et al. (2001). Lano, a novel LAP protein directly connected to MAGUK proteins in epithelial cells. J. Biol. Chem. 276, 32051-32055.

Tanentzapf, G., and Tepass, U. (2003). Interactions between the crumbs, lethal giant larvae and bazooka pathways in epithelial polarization. Nat. Cell Biol 5 $46-52$

Tepass, U., Tanentzapf, G., Ward, R., and Fehon, R. (2001). Epithelial cell polarity and cell junctions in Drosophila. Annu. Rev. Genet. 35, 747-784.

Tepass, U., Theres, C., and Knust, E. (1990). crumbs encodes an EGF-like protein expressed on apical membranes of Drosophila epithelial cells and required for organization of epithelia. Cell 61, 787-799.

Wodarz, A., Hinz, U., Engelbert, M., and Knust, E. (1995). Expression of crumbs confers apical character on plasma membrane domains of ectodermal epithelia of Drosophila. Cell 82, 67-76.

Yeaman, C., Grindstaff, K.K., Hansen, M.D., and Nelson, W.J. (1999). Cell polarity: versatile scaffolds keep things in place. Curr. Biol. 9, R515-517. 